\title{
Effect of metronidazole ophthalmic solution on corneal neovascularization in a rat model
}

\author{
Flor Diana Yokoay Claros-Chacaltana - Marcela Aldrovani • \\ Karina Kamachi Kobashigawa • Ivan Ricardo Martinez Padua • \\ Gisele Pereira Valdetaro • Alexandre Augusto Franchi de Barros Sobrinho • \\ Thaís Guimarães Morato Abreu · José Luiz Laus
}

Received: 29 November 2017 / Accepted: 13 April 2018/Published online: 26 April 2018

(C) Springer Science+Business Media B.V., part of Springer Nature 2018

\begin{abstract}
Purpose To evaluate the effect of metronidazole ophthalmic solutions on corneal neovascularization (CNV) in a rat model.

Methods A chemical burn was created in the right central cornea of 40 rats. Animals were randomized and distributed into four study groups ( $n=10$ rats per group) designated Met_0.1\%, Met_0.5\%, sham, and untreated groups. Chemical-burned corneas in the Met_0.1\% and Met_0.5\% groups received ophthalmic solutions of 0.1 and $0.5 \%$ metronidazole, respectively. Corneas in the sham group received phosphatebuffered saline (metronidazole diluent). All treated eyes received ophthalmic solution at intervals of $6 \mathrm{~h}$, for up to 30 days. Untreated corneas received no treatment. CNV was evaluated postinjury using corneal photographs at different evaluation time points. The main $\mathrm{CNV}$ outcome measures were: burn intensity, index of CNV, and percentage of vascularized corneal area. Five rats from each group were euthanized, on days 15 and 30; the samples were
\end{abstract}

F. D. Y. Claros-Chacaltana $(\bowtie) \cdot$ M. Aldrovani .

K. K. Kobashigawa · I. R. M. Padua · G. P. Valdetaro ·

A. A. F. de Barros Sobrinho - T. G. M. Abreu - J. L. Laus Ophthalmology Unit, Department of Veterinary Clinics and Surgery, School of Agricultural and Veterinarian Sciences (FCAV), São Paulo State University (UNESP), Via de acesso Prof. Paulo Donato Castellane s/n, Jaboticabal, SP CEP 14884-900, Brazil

e-mail: florclaros@hotmail.com collected for histological analyses. Differences with $P<0.05$ were considered significant.

Results CNV was observed in the eyes from day 7 postinjury. However, the indices of $\mathrm{CNV}$ for the Met_0.1\% and Met_0.5\% groups were smaller than those for the sham and untreated groups $(P<0.05)$. Furthermore, corneas treated with 0.1 or $0.5 \%$ metronidazole had smaller vascularized areas compared to control corneas. On histological study, the presence of blood vessels confirmed clinical outcomes.

Conclusions Regular instillation of 0.1 or $0.5 \%$ metronidazole had a significant inhibitory effect for $\mathrm{CNV}$ on chemical burns induced in a rat model.

Keywords Rats $\cdot$ Metronidazole $\cdot$ Cornea . Angiogenesis $\cdot$ Neovascularization

\section{Introduction}

Under normal homeostatic conditions, the cornea is transparent and avascular. However, the corneal angiogenic privilege is not absolute, in part because it results from the balance in the expression of antiangiogenic and pro-angiogenic molecules [1, 2]. When the expression of anti-angiogenic and proangiogenic molecules is altered, a pathological condition referred to as corneal neovascularization (CNV) may occur [2]. 
CNV alters visual acuity by inducing stromal edema, cellular infiltration, lipid deposition and scarring [2, 3]. It is estimated that up to 1.4 million people in the USA may have CNV [4]. CNV is a sightthreatening condition that may occur secondary to chemical burn, ischemia, inflammation, infection, limbal stem cell deficiency, degenerative disorders, post-corneal transplantation, and traumatic injuries, among others [5, 6]. It is also a potential complication of bacterial, parasitic, and viral infection [1].

Although the clinical relevance of $\mathrm{CNV}$ has been long recognized, its treatment is still challenging and associated with varying degrees of success [2, 6-8]. Current options for $\mathrm{CNV}$ include pharmacological approaches such as corticosteroids and non-steroidal anti-inflammatory agents, both of which may be associated with side effects. Other treatments include metalloproteinase inhibitors and monoclonal antibodies targeting vascular endothelial growth factors (VEGF), among others [6, 7]. Anti-VEGF antibodies, such as bevacizumab, ranibizumab and aflibercept, have a significant cost disadvantage despite the good results reported by different authors [9-11]. For patients in resource-poor countries, it is important to seek alternative, effective, and economically viable treatments for CNV.

Metronidazole is a low-cost drug used to treat infections caused by anaerobic bacteria and protozoans [12], to control odors in wounds [13], and to treat skin burns [14]. In ophthalmology, 0.1 and $0.5 \%$ metronidazole solutions have been used to treat keratitis [15-17]. Metronidazole also appears to have chemotherapeutic activity [18] and antioxidant effects [14]. More recently, there has been some evidence that metronidazole can control inflammation [19, 20] and modulate angiogenesis in non-ocular tissues [21, 22]. The aim of this study was to evaluate the angiogenic effect of metronidazole ophthalmic solutions on $\mathrm{CNV}$ in a rat model.

\section{Materials and methods}

Animals

Forty male Wistar rats (Rattus norvegicus), aged between 3 and 4 months, weighing $428.40 \pm 63.60 \mathrm{~g}$ (mean $\pm \mathrm{SD}$ ), were selected for the study. All the subjects were free of signs of corneal disorders [23].
The animals were kept in appropriate cages with an alternating light/dark cycle $(12 \mathrm{~h})$. They were fed a commercial pellet diet $(65.82 \%$ carbohydrate, $5.36 \%$ fiber, $21.0 \%$ proteins, and $4.96 \%$ fat) and received water ad libitum.

Corneal chemical burn models and treatment groups

Corneal inflammatory angiogenesis was induced through a protocol of punctual alkaline cauterization in the right eye [24]. All procedures were performed with animals under general [25] and topical anesthesia with $0.5 \%$ proxymetacaine eye drops (Alcon, São Paulo, Brazil). A circular disc of filter paper with a diameter of $3 \mathrm{~mm}$, saturated with a solution of $75 \%$ silver nitrate (Synth, Diadema, Brazil) and 25\% potassium nitrate (Dinâmica Contemporary Chemistry Ltda., Diadema, Brazil), was pressed onto the axial region of the cornea, for $20 \mathrm{~s}$ [24]. Excess silver and potassium nitrate were removed by rinsing the eye. To increase reproducibility, one investigator induced the chemical burn in all animals.

Following the chemical corneal burn, animals were randomized into four groups (Met_0.1\%, Met_0.5\%, sham, and untreated groups; $n=10$ rats per group), as shown in Fig. 1. All ophthalmic solutions used in the study, including the phosphate-buffered saline (PBS), the metronidazole diluent, were from single production lots manufactured by Ophthalmos S.A. (São Paulo, Brazil). Treatments started immediately postinjury and all instillations $(10 \mu \mathrm{L}$ of $0.1 \%$ metronidazole, $0.5 \%$ metronidazole, or PBS) were performed at regular intervals of $6 \mathrm{~h}$, for up to 30 days. Postinjury, the animals received tramadol hydrochloride $(5 \mathrm{mg}$ / $\mathrm{kg}$; Cristália, Itapira, Brazil) by the subcutaneous route every $8 \mathrm{~h}$, for 10 days [24].

Clinical examination of chemically burned corneas and image acquisition

Clinical examinations were performed after topical anesthesia ( $0.5 \%$ proparacaine; Alcon) and pupillary dilatation ( $1 \%$ tropicamide; Alcon). In addition, fluorescein eye stain (fluorescein sodium ophthalmic strips; Ophthalmos S.A., São Paulo, Brazil) was performed. Corneas were laterally and frontally photographed using digital equipment (TRC-50DX, Topcon, Japan) on days $0,2,7,12,15,18,21,26$, and 30 


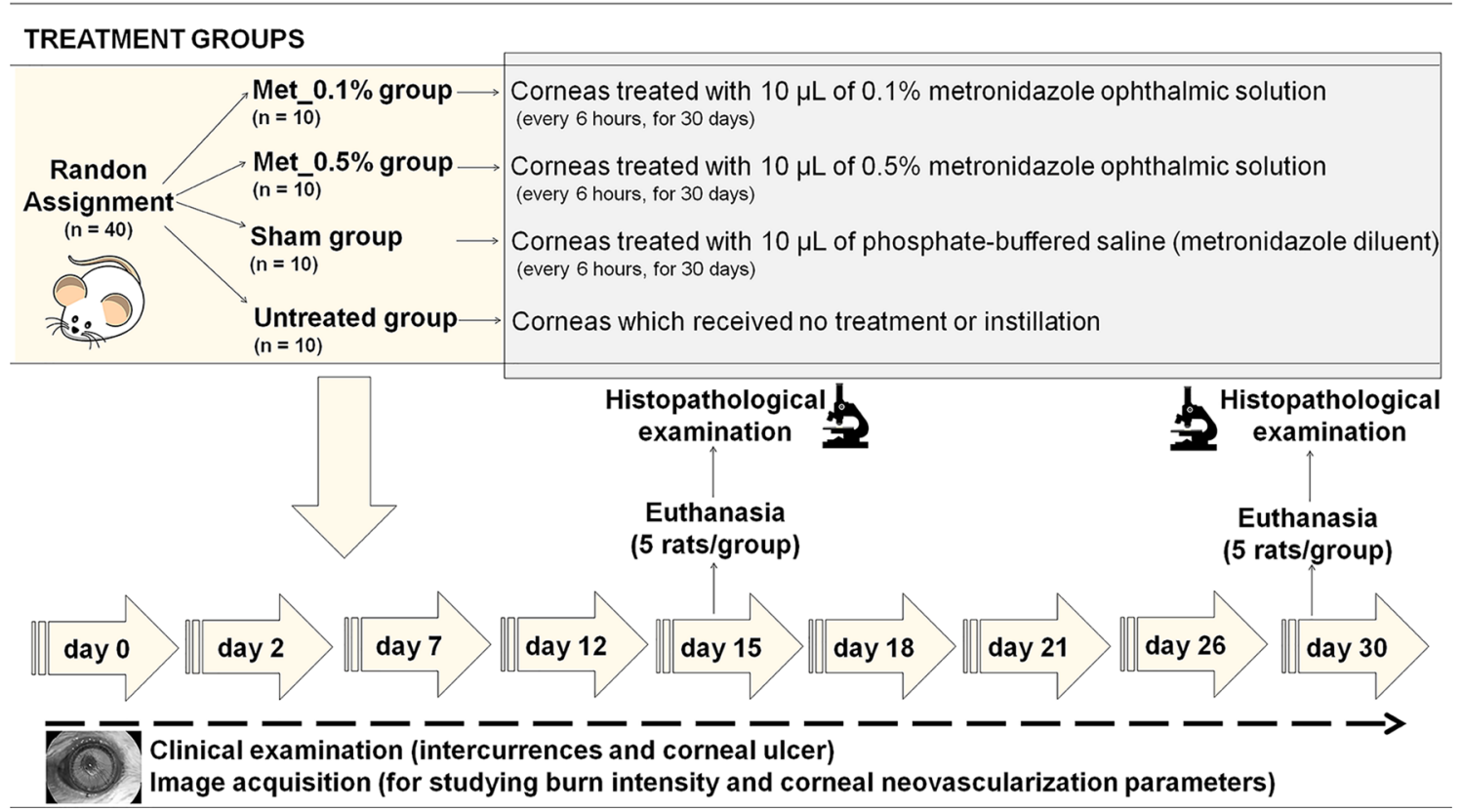

Fig. 1 Flowchart of the treatment groups compared in the study and simplified experimental design

$[8,24]$ and the images of CNV were evaluated by a blinded investigator.

Burn intensity and neovascularization parameters

Burn intensities were studied from lateral images [24]. They were scored based on the appearance of blisters and on the extent of their elevation from the corneal surface: 0 (no blister, not raised above corneal surface), 1 (a small blister that is slightly raised above the surface), 2 (a medium blister that is moderately raised above the surface), and 3 (a large blister) [26].

The CNV index and percentage of vascularized corneal area were studied from frontal profile images [24]. "The CNV index was scored between 0 and 3, with increments of 0.5 , per corneal quadrant using a grid system based on the centripetal extent of neovascular branch outgrowth from the corneoscleral limbus. Scores for each quadrant were then summed to obtain the CNV index (range from 0 to 12) for each eye at a given time point [27]".

The percentage of vascularized corneal area was calculated using a grid map containing 110 points, which were projected by the ImageJ software (National Institutes of Health, Bethesda, MD, USA) as previously described [24, 28]. Calculations were performed using the $(\mathrm{PV} \times 100) / \mathrm{PC}$ formula, where
PV represents the total number of points that touch the vessels and PC represents the total number of points that touch the non-vascularized corneal areas [24, 28].

To determine whether regression of $\mathrm{CNV}$ occurred during the follow-up time, we calculated the percentage differences between the final and the initial CNV area.

\section{Histopathological examination}

Five rats from each group, randomly selected, were euthanized on days 15 and 30 with intraperitoneal sodium thiopental (60 mg/kg; Cristália). The corneas were harvested, fixed in $10 \%$ buffered formaldehyde (Synth) and processed for routine inclusion in paraffin (Merck, Darmstadt, Germany). Sagittal sections of 4- $\mu \mathrm{m}$-thickness were stained with Masson trichrome staining and examined under a light microscope (Olympus BX53F microscope, Tokyo, Japan). The number of vessels per cornea section was manually counted at $40 \times$ magnification. The vascularization was histologically scored as 1 (minimal or close to negative vascularization), 2 (limited or focal vascularization in the sub-epithelial areas), 3 (intermediate between scores 2 and 4), or 4 (intense vascularization) [29]. 
Data analysis

All data were tested for statistical normality. Comparisons were performed using the Chi square test, the Kruskal-Wallis (KW) non-parametric test with Dunn's post hoc test, and the one-way analysis of variance (ANOVA) with Student-Newman-Keuls post hoc test. The odds ratio and Spearman's correlation were used as measures of association between the study variables. Differences with $P<0.05$ were considered significant (95\% confidence interval, $95 \%$ CI). All statistical analyses were performed using the MedCalc software (version 9.3.6.0, MedCalc, Mariakerke, Belgium).

\section{Results}

All corneas were fluorescein negative on day 0 postinjury. However, by day 2,1 cornea in the Met_0.1\% group (10\%), 4 in the Met_0.5\% group $(40 \%), 6$ in the sham group $(60 \%)$, and 7 corneas in the untreated group (70\%) were fluorescein positive. Corneal wound healing times were 7 days for the Met_0.1\% group, 12 days for the Met_0.5\% and untreated groups, and 18 days for the sham group (Chi square test contingency coefficient $=0.356$, $P<0.001)$.

All burn injury sites were opaque with a defined border (Fig. 2a), from days 2 to 12, corneas in all study groups presented blisters with varying degrees of elevation from the corneal surface (Fig. 2b-d). Corneas in the Met_0.1\% group showed less intense burns (maximum score of 1) than corneas in the sham and untreated groups (scores ranged from 0 to 3 ) $(P<0.05$, KW test with Dunn's post hoc test) (Table 1). The results in burn intensity observed between the Met_0.1\% and Met_0.5\% groups on day 12 of examination were not significantly different $(P>0.05)$. The results of the sham group were significantly different from those of the untreated group $(P<0.05)$ in all evaluation time points.

Formation of burn blister was not a univariate predictive factor for the development of corneal ulcer (OR 0.38 ; 95\% CI $0.21-0.69$ ). Only $14 \%$ of corneas that presented with burns with an intensity score above 1 developed ulceration.

CNV was observed in all groups on day 7. No association between the index of initial CNV (day 7) and the burn intensity was observed (Spearman's correlation $0.021 ; P=0.89$; 95\% CI $0.33-0.29$ ).

Statistical results related to the index of CNV are presented in Table 2. Both one-way ANOVA and KW tests were used to identify whether there was any significant difference in CNV between the groups, since the distributional assumptions required for parametric testing were not satisfied in all cases. ANOVA revealed that the mean indices of $\mathrm{CNV}$ for the Met_0.1\% and Met_0.5\% groups were significantly lower than those for the sham or untreated groups at all evaluation time points $(P<0.05$, ANOVA with Student-Newman-Keuls post hoc test), except on day 7. The KW test detected differences $(P<0.05)$ between groups only on days 12 and 15 .

Figure $3 a-d$ shows images of CNV observed in each study group. The Met_0.1\% and Met_0.5\% groups had a smaller area of CNV than sham and untreated groups (Fig. 4a). The CNV area was significantly smaller for the Met_0.1\% group than for the untreated and sham groups at all evaluation time points $(P<0.05$, KW with Dunn's post hoc test), except on day $7 \quad(P>0.05)$ (Fig. $4 \mathrm{~b}-\mathrm{h})$. The Met_0.5\% group results were significantly smaller than those of the sham and untreated groups on

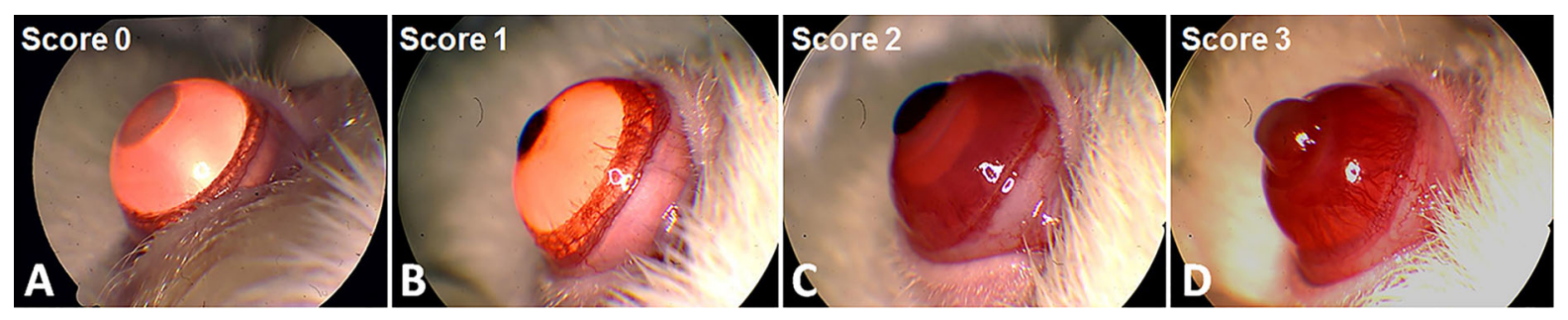

Fig. 2 Images of corneas according to the chemical burn intensity, i.e., the appearance of blisters and their elevation from the ocular surface, were scored as 0 (no blister, no raised above corneal surface) (a), 1 (a small blister that is slightly raised above the surface) (b), 2 (a medium blister that is moderately raised above the surface) (c), and 3 (a large blister) (d) 
18-30 days $(P<0.05)$. Boxplot charts revealed homogeneity in the variance of the data related to extensions in areas of $\mathrm{CNV}$ treated with 0.1 or $0.5 \%$ metronidazole.

Percentage differences between the final and the initial $\mathrm{CNV}$ areas were calculated. The $\mathrm{CNV}$ decreased by 60.90 and $38.91 \%$ in the Met_0.1\% and Met_0.5\% groups, respectively. In the sham and untreated groups, the CNV increased by 6.35 and $87.04 \%$, respectively.

No signs of irritation or complications were observed in the chemical-burned eyes that received 0.1 or $0.5 \%$ metronidazole for 15 or 30 days.

In the histopathological evaluation, corneas treated with 0.1 or $0.5 \%$ metronidazole exhibited fewer blood vessels than the sham or untreated corneas $(P<0.05$, KW with Dunn's post hoc test) (Fig. 5a, b). All corneas in the Met_0.1\% and Met_0.5\% groups were scored as 1 or 2, whereas more than half of the corneas in the sham and untreated groups were scored as 3 or 4 (Fig. 5c, d). Figure 5e-h shows images of chemicalburned corneas that were histologically scored as 1,2 , 3 , and 4 according to their vascularization. The only pathological change observed in corneas scored as 1 or 2 was the presence of blood vessels in the anterior stroma proximal to the epithelium (Fig. 5e-f). Approximately, $30 \%$ of corneas that were scored as 3 or 4 , in addition to blood vessels, had mild-tomoderate inflammatory infiltrate and focal fibroblastic activity in the central anterior stroma (Fig. $5 \mathrm{~g}$ ). In the untreated group, $20 \%$ of corneas scored as 4 showed neovascularization, disorganization of stromal collagen fibers and thickening of the central epithelium (Fig. 5h).

\section{Discussion}

$\mathrm{CNV}$ is a clinical condition that, when left untreated, causes significant visual deficit [5]. Current therapies for inhibiting angiogenesis in vascularized corneas are associated with a variety of systemic side effects [10]. Moreover, some treatments have a significant cost disadvantage and are inaccessible to some part of the population in resource-poor countries. To benefit and treat patients from these countries, it is necessary to investigate an anti-angiogenic potential of more affordable drugs. Metronidazole, a nitroimidazole derivative $[12,20,30]$, is inexpensive; in some 


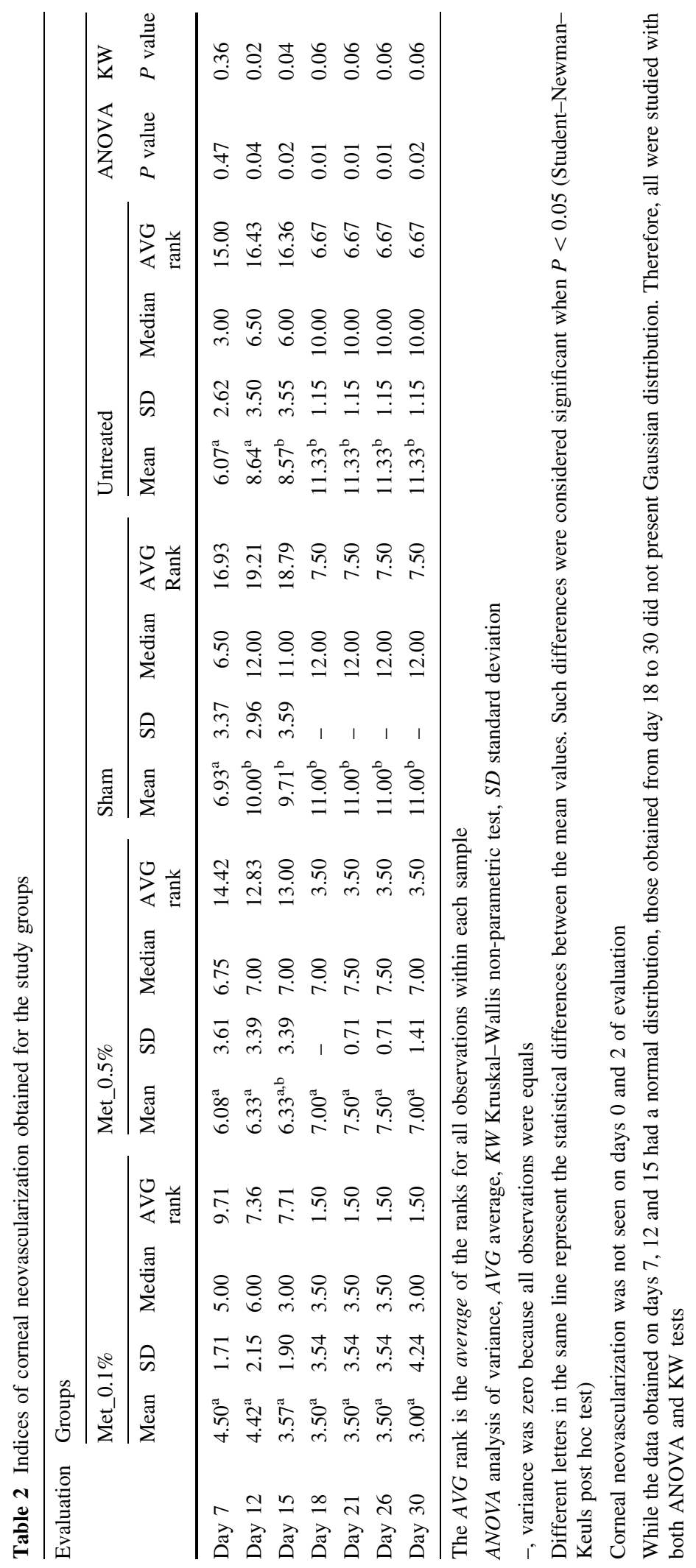




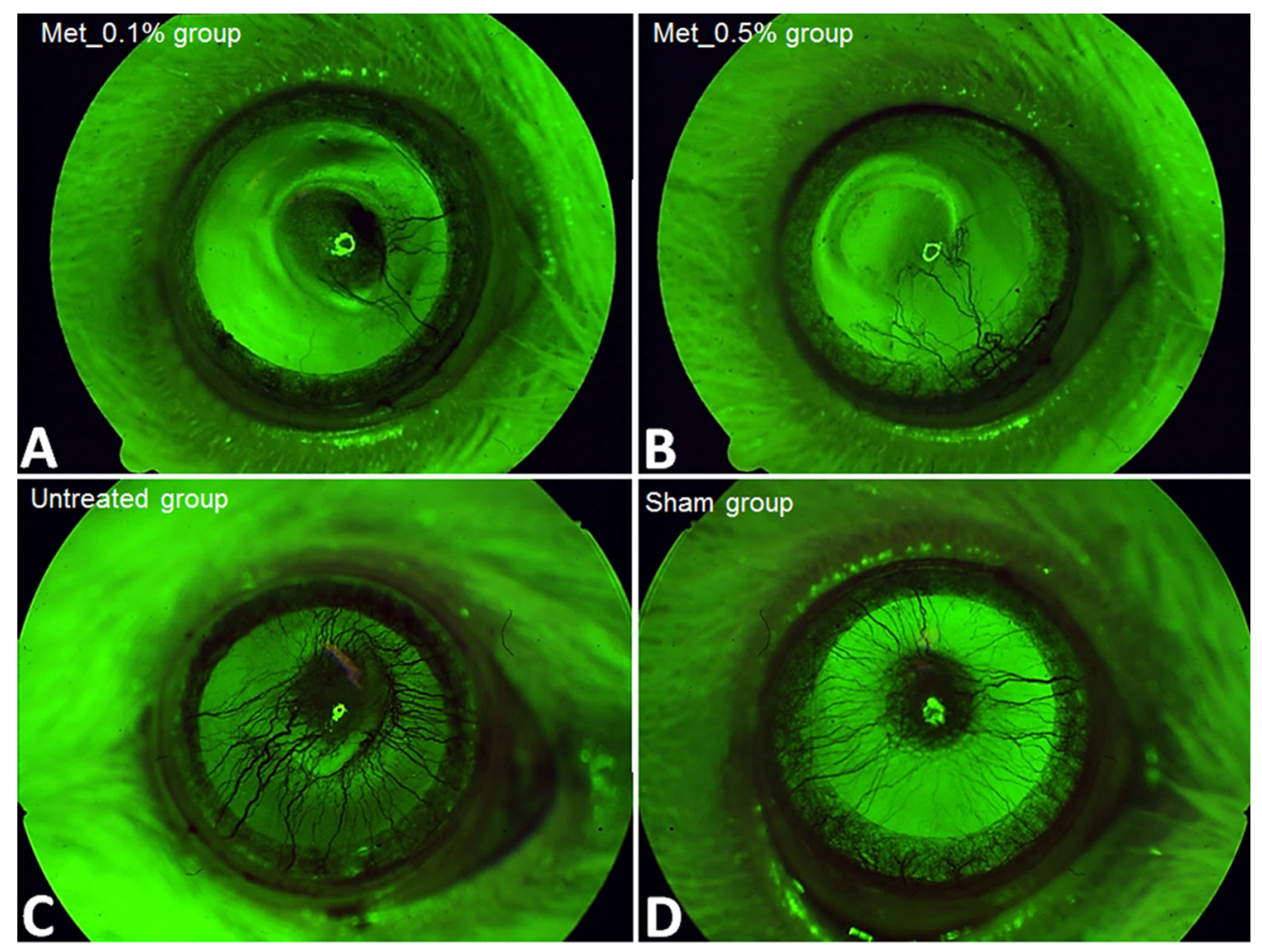

Fig. 3 Panel containing representative images of CNV (red-free filter) observed in each study group. Images a and $\mathbf{b}$ show corneas in the Met_0.1\% and Met_0.5\% groups with smaller vascularized areas than corneas in the untreated and sham groups (c, d)

countries, such as Brazil, it is available free of charge from the National Public Health System.

Nishimuta and Ito [19] reported that side effects related to topical metronidazole are lower than those of corticosteroids. Also, other authors who used $0.5 \%$ metronidazole to treat keratitis also did not report any side effects [31]. In addition, toxicological studies have shown that topical use of nitroimidazole derivatives does not cause cell mutation [19]. In contrast, systemic use of nitroimidazole derivatives has been associated with long-term side effects [20].

Healing properties have been attributed to metronidazole $[14,21,32]$. These properties arise from metronidazole's ability to promote contraction of myofibroblasts, stimulate the proliferation of epithelial cells, inhibit local inflammation, and reduce bacterial load [14, 20, 21, 32]. In fact, our results on the occurrence of corneal ulcer and times of corneal epithelial wound healing suggest that metronidazole may enhance healing, depending on the concentration used. Corneal ulcer in the Met_0.1\% group healed at 7 days postinjury, while corneal ulcer in the Met_0.5\% group healed at 12 days postinjury. It is important to note that in this study, corneal ulceration was not strongly influenced by the formation of burn blisters, as shown by the odds ratio calculation.

Evidence suggests that metronidazole has antioxidant and immunosuppressive effects $[14,20]$ and that it can modulate tissue angiogenesis [21, 22, 33]. However, in the latter respect, the results from studies by different authors are controversial. While some authors have observed that metronidazole has proangiogenic action [21], others have reported that it inhibits angiogenesis [22, 33]. Sampaio et al. [21] observed that metronidazole contributed to healing in second intention wounds and stimulated collagen production and angiogenesis. In contrast, Pezo [33], studying the chicken chorioallantoic membrane, found that metronidazole inhibited new blood vessels formation. Michalska et al. [22] observed that metronidazole, when combined with clindamycin, inhibited neovascularization in benign gynecological tumors. The clinical and histopathological findings in this study agree with the results of studies that associated metronidazole with inhibition of tissue angiogenesis. 

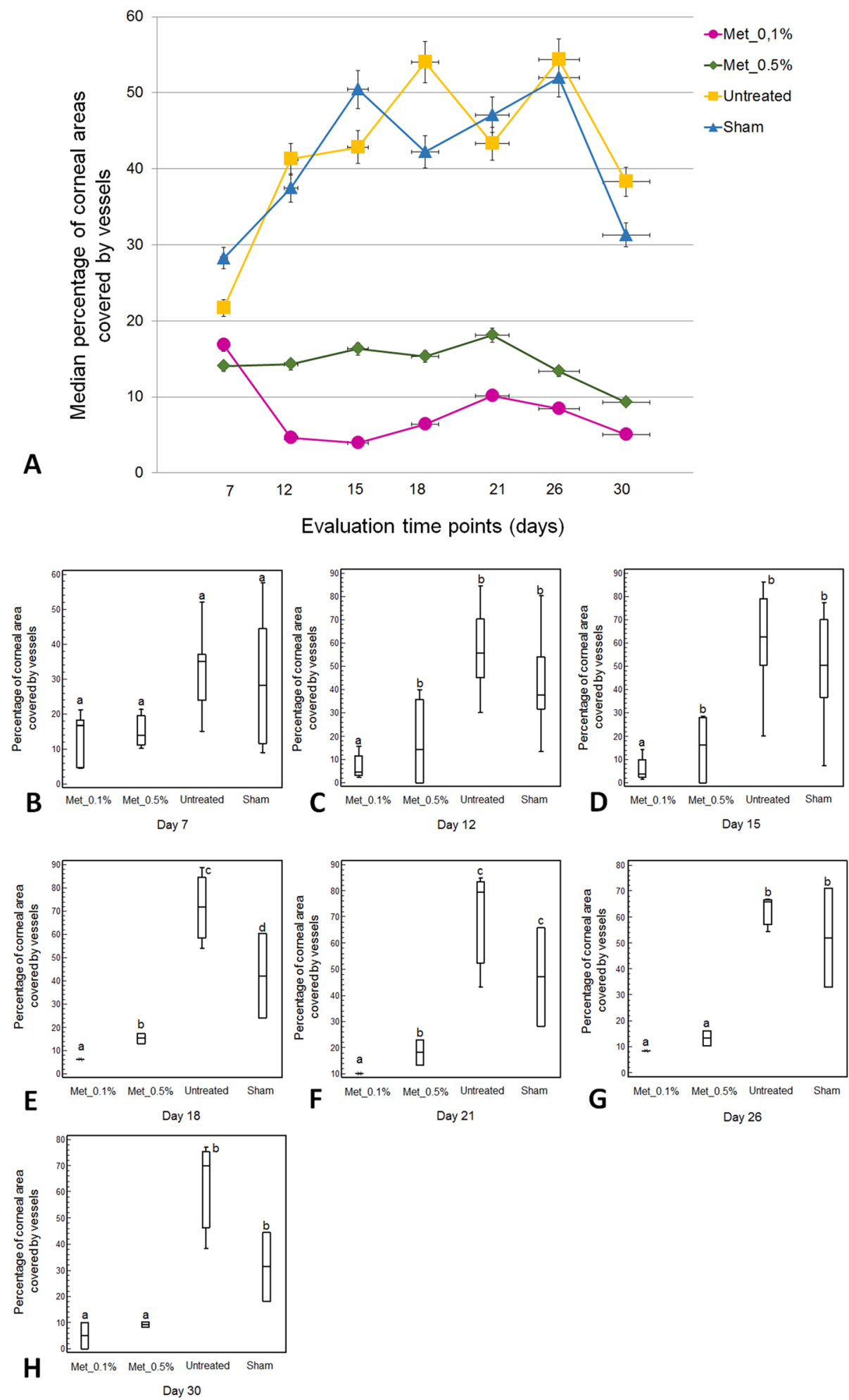
4Fig. 4 Extension of Corneal neovascularization. a Corresponds to a line chart showing the differences in percentage of vascularized corneal area between the study groups (bh) correspond to boxplot charts showing the extent of vascularized corneal area in each study group on days 7,12 , $15,18,21,26$, and 30 postinjury. Different letters represent the statistical differences $(P<0.05$, Kruskal-Wallis test with Dunn's post hoc test)

The chemical burn model used in this study is known to induce diffuse formation of vessels in a large region of the cornea [7, 24, 34]. The clinical parameters of CNV revealed that the pattern of initial vessel development was identical for all studied groups, even though, some differences in the evolution of CNV were detected only from day 12 . We believe that the differences in the evolution of CNV between the study groups result from the treatment protocols used. The clinical and histopathological findings of this work strongly suggest that metronidazole inhibits growth of vessels and promotes regression of $\mathrm{CNV}$ in a rat model. After 30 days of treatment, there was a significant reduction of vascularization in corneas treated with 0.1 or $0.5 \%$ metronidazole. The antiangiogenic effects of metronidazole have apparently been consistent, since the boxplots (Fig. 3c-i) revealed homogeneity (i.e., low dispersion) in the variance of the data related to extensions of vascularized areas in corneas treated with 0.1 or $0.5 \%$ metronidazole. CNV area results in this study are similar to those observed by Gal-Or et al. [11] when they studied the effects of aflibercept and bevacizumab on $\mathrm{CNV}$.

The therapeutic effects of a drug may vary depending on the dosage and the protocol of administration [35-37]. In the present study, $0.1 \%$ metronidazole was more effective than $0.5 \%$ metronidazole in promoting corneal healing and inhibiting CNV. A possible explanation for differences in outcomes between corneas in the Met_0.1\% and Met_0.5\% groups is that the action of metronidazole on the corneal tissue can be dose dependent. Drugs used at higher or lower concentrations than an optimal dose may be less effective or ineffective in enhancing corneal healing and inhibiting neovascularization [37]. However, it is not possible to affirm that $0.1 \%$ is the optimum concentration of metronidazole since dose-response curves were not constructed. The concentrations evaluated herein are commercially available and are widely used to treat some kinds of infectious keratitis $[16,31]$. The possibility that $0.5 \%$ metronidazole was less effective in treating $\mathrm{CNV}$ because it was irritating to the cornea was discarded, as the eyes show no sign of irritation.

The mechanisms by which metronidazole acts on corneal angiogenesis were not object of this investigation. To our knowledge, there is no study on whether metronidazole exacerbates the expression of antiangiogenic molecules reduces the production of proangiogenic molecules, or both. Some authors have suggested that it acts by reducing inflammation $[19,20]$. Additionally, metronidazole reduces the generation of free radicals by neutrophils [38, 39], attenuating the oxidative stress associated with inflammation and CNV [40]. This hypothesis needs to be further investigated.

In this study, as in many others that evaluated drug effects on $\mathrm{CNV}$, immunohistochemistry was not performed to differentiate blood vessels from lymphatic vessels $[3,6-8,11]$. Chemical burn to the cornea generates a non-specific inflammatory response that causes lymphangiogenesis [41]. Thus, metronidazole, by reducing the inflammation, can also have inhibited lymphatic vessel growth in the chemical-burned corneas. However, estimation of the effect of metronidazole on $\mathrm{CNV}$ was not compromised by a possible action of the drug on lymphatic vessels, mainly because the histopathological examination confirmed fewer blood vessels in the corneas of the Met_0.1\% and Met_0.5\% groups. In addition, neovessels and lymphatic vessels differ in sprout and distribution patterns [42], which also allow us to state that metronidazole has acted on CNV. Following inflammatory stimuli, pathological lymphatic vessels sprout predominantly from the nasal region of the corneal limbus, occupying a corneal area much less than blood vessels [42].

An interesting finding from this study was that the placebo (PBS) was not inert and can introduce bias against tested ophthalmic products. In agreement with this, the literature is replete with clinical trials where placebo procedures were assumed to be inert [43-45] but were not. In this study, the corneas in the sham group developed less intense burn injuries than the corneas in the untreated group. We suspect that the placebo attenuated burn injury by providing moisture to the ocular surface (lubrication) as well as by removing pathogens, allergens, and cell degradation 

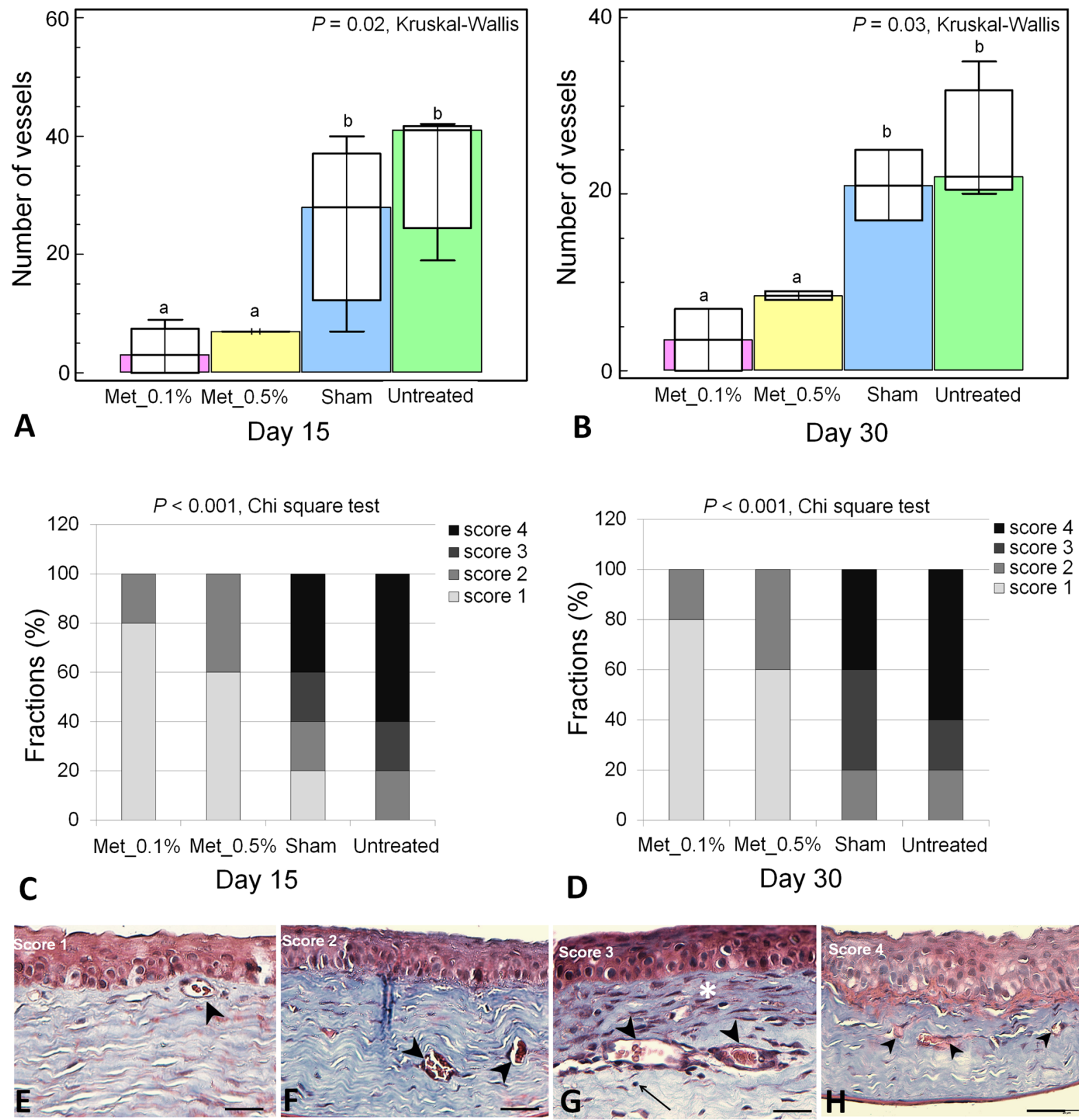

Fig. 5 Histopathological examination of vascularized corneas. a, b Correspond to combined boxplot and bar charts (of median values, with interquartile range error bars) showing the numbers of vessels counted in corneas of each study group on days 15 and 30 of examination. Note that corneas in the Met_0.1\% and Met_0.5\% groups showed smaller vessels than the untreated and sham groups. Different letters represent the statistical differences $(P<0.05$, Kruskal-Wallis test with Dunn's post hoc test). c, d Corresponds to the stacked bar graphs showing

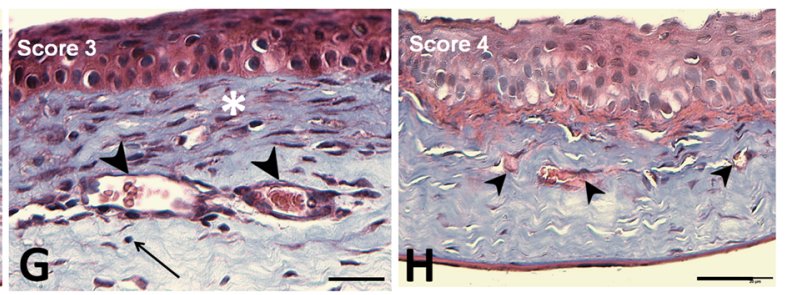

percentages of corneas scored 1, 2, 3, and 4 for each study group on days 15 and 30 postinjury. e Corresponds to the photomicrography according to the vascularization that was histologically scored 1 . f Score 2 . g Score 3 . h Score 4. Arrow heads indicate the vessels, the black arrow points to an inflammatory cell, and the asterisk indicates a focus of fibroblastic activity. Note that the epithelium of the cornea scored as 4 is thickened. Bar $=20 \mu \mathrm{m}$ 
products [46]. Previous studies have shown that the use of ocular lubricants causes a transient increase in thickness of the tear film [47], which dilutes molecules that stimulate the appearance or progression of superficial corneal damage.

As a limitation, in this study, our material was not sufficient to quantify the expression of anti-angiogenic and pro-angiogenic molecules. To ensure that the sample size would give adequate power for the histopathological evaluation of $\mathrm{CNV}$, all corneas collected from rats were paraffin embedded and cross sectioned; in this way, the expressions of VEGF and other molecules are difficult to assess because they are highly localized. Furthermore, tissue section immunohistochemical studies with manual scoring are not a quantitative measurement but rather a qualitative assessment [48]. Expression of anti-angiogenic and pro-angiogenic molecules in chemical-burned corneas treated with metronidazole will be evaluated in future using polymerase reaction chain, enzyme-linked immunosorbent assay, and western blot.

In conclusion, regular instillation of 0.1 or $0.5 \%$ metronidazole inhibits and decreases the progression of $\mathrm{CNV}$ on chemical burns induced in a rat model. Additional research is required to determine the molecular mechanism of action of metronidazole ophthalmic solutions on corneal angiogenesis, as well as to establish an optimum dose and possible side effects.

Acknowledgements The authors would like to thank Ophthalmos S.A. (São Paulo, Brazil) for donating the ophthalmic solutions used in the research. We would also like to thank Professors Kenia Cardoso Bícego and Joaquim Mansano Garcia for allowing us to use of the Vivarium; Professor Annelise Camplesi, Mr. Euclides Roberto Secato, Ph.D. Edwin Gutierrez Rodriguez, master Karina Herencia Bueno and Maria Lucia Correal for their help in handling animals; Carol Scarpellini and Vivian Biancardin for donation of the rats and Fausto Marinho Neto for the help in making histopathological sections.

Funding This study was funded by the National Council for Scientific and Technological Development (CNPq Proc. 300833/2010-5), the Sao Paulo Research Foundation (FAPESP Proc. 2009/51773-4 and 2012/17308-5), and the Coordination for the Improvement of Higher Education Personnel (CAPES Proc. 88881.133761/2016-01).

\section{Compliance with ethical standards}

Conflict of interest The authors declare that they have no conflict of interest.
Ethical approval All procedures performed in the study were in accordance with the ARVO Statement for Use of Animals in Ophthalmic Vision and Research. The ethical principles established by the National Institutes of Health Guide for the Care and Use of Laboratory Animals (NIH Publications No. 8523, revised 2011) were followed. The research protocol was approved by the Ethics Committee on Animal Use (Protocol No. 06174/14) of FCAV/Unesp, Jaboticabal.

\section{References}

1. Chang JH, Garg NK, Lunde E, Han KY, Jain S, Azar DT (2012) Corneal neovascularization: an anti-VEGF therapy review. Surv Ophthalmol 57(5):415-429. https://doi.org/10. 1016/j.survophthal.2012.01.007

2. Stevenson W, Cheng S, Dastjerdi MH, Ferrari G, Dana R (2012) Corneal neovascularization and the utility of topical VEGF inhibition: ranibizumab (Lucentis) vs bevacizumab (Avastin). Ocul Surf 10(2):67-83. https://doi.org/10.1016/j. jtos.2012.01.005

3. Onder HI, Erdurmus M, Bucak YY, Simavli H, Oktay M, Kukner AS (2014) Inhibitory effects of regorafenib, a multiple tyrosine kinase inhibitor, on corneal neovascularization. Int J Ophthalmol 7(2):220. https://doi.org/10.3980/ j.issn.2222-3959.2014.02.06

4. Lee P, Wang CC, Adamis AP (1998) Ocular neovascularization: an epidemiologic review. Surv Ophthalmol 43:245-269. https://doi.org/10.1016/S00396257(98)00035-6

5. Steger B, Romano V, Kaye SB (2016) Corneal indocyanine green angiography to guide medical and surgical management of corneal neovascularization. Cornea 35(1):41-45. https://doi.org/10.1097/ICO.0000000000000683

6. Schaap-Fogler M, Bahar I, Rephaeli A, Dahbash M, Nudelman A, Livny E, Barliya T, Nisgav Y, Livnat T (2017) Effect of histone deacetylase inhibitor, butyroyloxymethyldiethyl phosphate (AN-7), on corneal neovascularization in a mouse model. J Ocul Pharmacol Ther 33(6):480-486. https://doi.org/10.1089/jop.2016.0017

7. Hosseini H, Nejabat M, Mehryar M (2007) Bevacizumab inhibits corneal neovascularization in an alkali burn induced model of corneal angiogenesis. Clin Exp Ophthalmol 35:745-748. https://doi.org/10.1111/j.1442-9071.2007. 01572.x

8. Kang S, Chung SK (2010) The effect of subconjunctival combined treatment of bevacizumab and triamcinolone acetonide on corneal neovascularization in rabbits. Cornea 29(2):192-196. 0b013e3181b1c82f

9. Bock F, Onderka J, Dietrich T, Bachmann B, Kruse FE, Paschke M, Grit Z, Cursiefen C (2007) Bevacizumab as a potent inhibitor of inflammatory corneal angiogenesis and lymphangiogenesis. Investig Ophthalmol Vis Sci 48:2545-2552. https://doi.org/10.1167/iovs.06-0570

10. Ferrari G, Dastjerdi MH, Okanobo A, Cheng SF, Amparo F, Nallasamy N, Dana R (2013) Topical ranibizumab as a treatment of corneal neovascularization. Cornea 32(7):992-997. https://doi.org/10.1097/ICO. 0b013e 3182775f8d 
11. Gal-Or O, Livny E, Sella R, Nisgav Y, Weinberger D, Livnat T, Bahar I (2016) Efficacy of subconjunctival aflibercept versus bevacizumab for prevention of corneal neovascularization in a rat model. Cornea 35(7):991-996. https://doi.org/10.1097/ICO.0000000000000849

12. Pérez-Trallero E, Iglesias L (2003) Tetracyclines, sulfonamides and metronidazole. Enferm Infecc Microbiol Clin 21(9):520-529. https://doi.org/10.1016/S0213005X(03)72999-1

13. Castro DLV, Santos VLCG (2015) Odor management in fungating wounds with metronidazole. J Hosp Palliat Nurs 17(1):73-79. 0000000000000125

14. Rao CM, Ghosh A, Raghothama C et al (2002) Does metronidazole reduce lipid peroxidation in burn injuries to promote healing? Burns 28(5):427-429. https://doi.org/10. 1016/S0305-4179(01)00126-7

15. Van Der Bijl P, Van Eyk AD, Seifart HI et al (2004) In vitro transcorneal penetration of metronidazole and its potential use as adjunct therapy in acanthamoeba keratitis. Cornea 23:386-389.

https://doi.org/10.1097/00003226200405000-00014

16. Lima AAS (2006) Formulações Magistrais em oftalmologia. Cultura Medica, Rio de Janeiro

17. Kodym A, Kaczy-Ska-Dyba E, Kubiak B, Kukula H (2011) Technology of eye drops containing metronidazole. Acta Pol Pharm 68(1):109-114

18. Qian Y, Zhang H, Zhang H, Xu Q, Zhao J, Zhu HL (2010) Synthesis, molecular modeling, and biological evaluation of cinnamic acid metronidazole ester derivatives as novel anticancer agents. Bioorg Med Chem 18:4991-4996. https://doi.org/10.1016/j.bmc.2010.06.003

19. Nishimuta K, Ito Y (2003) Effects of metronidazole and tinidazole ointments on models for inflammatory dermatitis in mice. Arch Dermatol Res 294(12):544-551. https://doi. org/10.1007/s00403-002-0381-4

20. Ypsilantis E, Carapeti E, Chan S (2015) The use of topical $10 \%$ metronidazole in the treatment of non-healing pilonidal sinus wounds after surgery. Int $\mathrm{J}$ Colorectal Dis 3(31):765-767. https://doi.org/10.1007/s00384-015-2269-8

21. Sampaio CPP, Biondo-Simões MLP, Trindade LCT, Farias RE, Pierin RJ, Martins RC (2009) Inflammatory alterations provoked by metronidazole in wounds: an experimental study in rats. J Vasc Bras 8(3):232-237. https://doi.org/10. 1590/S1677-54492009000300008

22. Michalska M, Palatyńska-Ulatowska A, Palatyński A, Mirowski M, Kaplinska K, Nawrot-Modranka J, Lazarenkow A (2011) Influence of antibiotic therapy on the level of selected angiogenic factors in patients with benign gynecologic tumors-preliminary report. Pharmazie 66(8):619-622. https://doi.org/10.1691/ph.2011.0902

23. Claros-Chacaltana FDY, Kobashigawa KK, Padua IRM, Valdetaro GP, Aldrovani M, Laus JL (2017) Persistent pupillary membrane in Wistar laboratory rats (Rattus Norvegicus, Albinus Variation, Wistar). Cienc Rural 47(3):1-6. https://doi.org/10.1590/0103-8478cr20160421

24. Claros-Chacaltana FDY, Kobashigawa KK, Padua IRM, Valdetaro GP, Aldrovani M, Laus JL (2017) Corneal angiogenesis based on different protocols of alkaline cauterization in murine models. Acta Bras Cir 32(8):607-616. https://doi.org/10.1590/s0102-865020170080000002
25. Mohan RR, Stapleton WM, Sinha S, Netto MV, Wilson SE (2008) A novel method for generating corneal haze in anterior stroma of the mouse eye with the excimer laser. Exp Eye Res 86:235-240. https://doi.org/10.1016/j.exer.2007. 10.014

26. Mahoney JM, Waterbury LD (1985) Drug effects on the neovascularization response to silver nitrate cauterization of the rat cornea. Curr Eye Res 4(5):531-535. https://doi.org/ $10.3109 / 02713688508999984$

27. Yamada J, Dana MR, Sotozono C, Kinoshita S (2003) Local suppression of IL-1 by receptor antagonist in the rat model of corneal alkali injury. Exp Eye Res 76(2):161-167. https:// doi.org/10.1016/S0014-4835(02)00293-2

28. Bostrom M, Kalm M, Karlsson N, Erkenstam NH, Blomgren K (2013) Irradiation to the young mouse brain caused long-term, progressive depletion of neurogenesis but did not disrupt the neurovascular niche. J Cereb Blood Flow Metab 33:935-943. https://doi.org/10.1038/jcbfm.2013.34

29. Ozdemir O, Altintas O, Altintas L (2014) Comparison of the effects of subconjunctival and topical anti-VEGF therapy (bevacizumab) on experimental corneal neovascularization. Arq Bras Oftalmol 77(4):209-213. https://doi.org/10.5935/ 0004-2749.20140054

30. Löfmark S, Edlund C, Nord CE (2010) Metronidazole is still the drug of choice for treatment of anaerobic infections. Clin Infect Dis 50(1):S16-S23. https://doi.org/10.1086/647939

31. Yeung EY, Huang SC, Tsai RJ (2002) Acanthamoeba keratitis presenting as dendritic keratitis in a soft contact lens wearer. Chang Gung Med J 25(3):201-206

32. Nicholson TJ, Armstrong D (2004) Topical metronidazole (10 percent) decreases posthemorrhoidectomy pain and improves healing. Dis Colon Rectum 47(5):711-718. https://doi.org/10.1007/s10350-003-0129-z

33. Pezo C (2012) Efecto angiogénico de Metronidazol y Alfa Bisabolol en membrana alantocoriónica de pollo, un modelo in vivo en angiogénesis experimental. Bio noticias 1:63-64

34. Sener E, Yuksel N, Yildiz DK (2011) The impact of subconjunctivally injected EGF and VEGF inhibitors on experimental corneal neovascularization in rat model. Curr Eye Res 36:1005-1013. https://doi.org/10.3109/02713683. 2011.601840

35. Buttgereit F, da Silva JAP, Boers M, Burmester G-R, Cutolo M, Jacobs J, Kirwan J, Köhler L, van Riel P, Vischer T, Bijlsma JWJ (2002) Standardised nomenclature for glucocorticoid dosages and glucocorticoid treatment regimens: current questions and tentative answers in rheumatology. Ann Rheum Dis 61:718-722. https://doi.org/10.1136/ard. 61.8.718

36. Laato M, Niinikoski J, Lebel L, Gerdin B (1986) Stimulation of wound healing by epidermal growth factor. A dosedependent effect. Ann Surg 203(4):379-381. https://doi. org/10.1097/00000658-198604000-00007

37. Mathers WD, Sherman M, Fryczkowski A, Jester JV (1989) Dose-dependent effects of epidermal growth factor on corneal wound healing. Invest Ophthalmol Vis Sci 30(11):2403-2406

38. Akamatsu H, Oguchi M, Nishijima S, Asada Y, Takahashi M, Ushijima T, Niwa Y (1990) The inhibition of free radical generation by human neutrophils through the synergistic effects of metronidazole with palmitoleic acid: a possible mechanism of action of metronidazole in rosacea and acne. 
Arch Dermatol Res 282:449-454. https://doi.org/10.1007/ BF00402621

39. Mcclellan KJ, Noble S (2000) Topical metronidazole. Am J Clin Dermatol 1(3):191-199. https://doi.org/10.2165/ 00128071-200001030-00007

40. Dong A, Xie B, Shen J, Yoshida T, Yokoi K, Hackett SF, Campochiaro PA (2009) Oxidative stress promotes ocular neovascularization. J Cell Physiol 219(3):544-552. https:// doi.org/10.1002/jcp. 21698

41. Chauhan SK, Dohlman TH, Dana R (2014) Corneal lymphatics: role in ocular inflammation as inducer and responder of adaptive immunity. J Clin Cell Immunol 5:1000256. https://doi.org/10.4172/2155-9899.1000256

42. Shi W, Ming C, Liu J, Wang T, Gao H (2011) Features of corneal neovascularization and lymphangiogenesis induced by different etiological factors in mice. Graefes Arch Clin Exp Ophthalmol 249:55-67. https://doi.org/10.1007/ s00417-010-1442-6

43. Birch S (2006) A review and analysis of placebo treatments, placebo effects, and placebo controls in trials of medical procedures when sham is not inert. J Altern Complement Med 12(3):303-310. https://doi.org/10.1089/acm.2006.12. 303
44. Finniss DG, Kaptchuk TJ, Miller F, Benedetti F (2010) Placebo effects: biological. Clinical and ethical advances. Lancet 375(9715):686-695. https://doi.org/10.1016/S01406736(09)61706-2

45. Gupta U, Verma M (2013) Placebo in clinical trials. Perspect Clin Res 4(1):49-52. https://doi.org/10.4103/22293485.106383

46. Margo CE (1999) The placebo effect. Surv Ophthalmol 44(1):31-44. https://doi.org/10.1016/S00396257(99)00060-0

47. Dienes L, Kiss HJ, Perényi K, Szepessy Z, Nagy ZZ, Barsi A, Acosta MC, Gallar J, Kovács I (2015) The effect of tear supplementation on ocular surface sensations during the interblink interval in patients with dry eye. PLoS ONE 10(8):e0135629. https://doi.org/10.1371/journal.pone. 0135629

48. Chung JY, Braunschweig T, Hong SM, Kwon DS, Eo SH, Cho H, Hewitt Stephen M (2014) Assessment of vascular endothelial growth factor in formalin fixed, paraffin embedded colon cancer specimens by means of a well-based reverse phase protein array. Proteome Sci 12:27. https://doi. org/10.1186/1477-5956-12-27 